\title{
FEKETE-SZEGÖ INEQUALITY FOR ANALYTIC AND BI-UNIVALENT FUNCTIONS SUBORDINATE TO CHEBYSHEV POLYNOMIALS
}

\author{
FERAS YOUSEF, B. A. FRASIN, AND TARIQ AL-HAWARY
}

\begin{abstract}
In the present paper, a new subclass of analytic and bi-univalent functions by means of Chebyshev polynomials is introduced. Certain coefficient bounds for functions belong to this subclass are obtained. Furthermore, the Fekete-Szegö problem in this subclass is solved.
\end{abstract}

\section{INTRODUCTION}

The classical Chebyshev polynomials of degree $n$ of the first and second kinds, which are denoted respectively by $T_{n}(t)$ and $U_{n}(t)$, have generated a great deal of interest in recent years. These orthogonal polynomials, in a real variable $t$ and a complex variable $z$, have played an important role in applied mathematics, numerical analysis and approximation theory. For this reason, Chebyshev polynomials have been studied extensively, see $[8,10$, 16]. In the study of differential equations, the Chebyshev polynomials of the first and second kinds are the solution to the Chebyshev differential equations

$$
\left(1-t^{2}\right) y^{\prime \prime}-t y^{\prime}+n^{2} y=0
$$

and

$$
\left(1-t^{2}\right) y^{\prime \prime}-3 t y^{\prime}+n(n+2) y=0,
$$

respectively. The roots of the Chebyshev polynomials of the first kind are used as nodes in polynomial interpolation and the monic Chebyshev polynomials minimize all norms among monic polynomials of a given degree. For a brief history of Chebyshev polynomials of the first and second kinds and their applications, the reader is referred to [19, 22].

A classical result of Fekete and Szegö [13] determines the maximum value of $\left|a_{3}-\eta a_{2}^{2}\right|$, as a non-linear functional of the real parameter $\eta$, for the class of normalized univalent functions

$$
f(z)=z+a_{2} z^{2}+a_{3} z^{3}+\cdots .
$$

There are now several results of this type in the literature, each of them dealing with $\left|a_{3}-\eta a_{2}^{2}\right|$ for various classes of functions defined in terms of subordination (see e.g., $[1,20]$ ). Motivated by the earlier work of Dziok et al. [10], the main focus of this work is to utilize the Chebyshev polynomials expansions to solve Fekete-Szegö problem for certain subclass of bi-univalent functions (see, for example, $[5,6,7,14]$ ).

2010 Mathematics Subject Classification. Primary 30C45; Secondary 30C50.

Key words and phrases. Analytic functions, bi-univalent functions, Fekete-Szegö problem, Chebyshev polynomials, coefficient bounds, subordination. 
This paper is divided into three sections with this introduction being the first. In Section 2, we define the class of analytic and bi-univalent functions $\mathscr{B}_{\Sigma}(\lambda, \mu, t)$ using the generating function for the Chebyshev polynomials of the second kind, and we also discuss some other definitions and results. Section 3 is devoted to solve problems concerning the coefficients of functions in the class $\mathscr{B}_{\Sigma}(\lambda, \mu, t)$. Section 4 is the main part of the paper, we find the sharp bounds of functionals of Fekete-Szegö type.

\section{Definitions AND PRELIMINARIES}

Let $\mathscr{A}$ denote the class of functions of the form:

$$
f(z)=z+\sum_{n=2}^{\infty} a_{n} z^{n},
$$

which are analytic in the open unit disk $\mathbb{U}=\{z \in \mathbb{C}:|z|<1\}$. Further, by $\mathscr{S}$ we shall denote the class of all functions in $\mathscr{A}$ which are univalent in $\mathbb{U}$.

Given two functions $f, g \in \mathscr{A}$. The function $f(z)$ is said to be subordinate to $g(z)$ in $\mathbb{U}$, written $f(z) \prec g(z)$, if there exists a Schwarz function $\omega(z)$, analytic in $\mathbb{U}$, with

$$
\omega(0)=0 \text { and }|\omega(z)|<1 \text { for all } z \in \mathbb{U},
$$

such that $f(z)=g(\omega(z))$ for all $z \in \mathbb{U}$. Furthermore, if the function $g$ is univalent in $\mathbb{U}$, then we have the following equivalence (see [17] and [23]):

$$
f(z) \prec g(z) \Leftrightarrow f(0)=g(0) \text { and } f(\mathbb{U}) \subset g(\mathbb{U}) .
$$

The Koebe one-quarter theorem [9] asserts that the image of $\mathbb{U}$ under each univalent function $f$ in $\mathscr{S}$ contains a disk of radius $\frac{1}{4}$. According to this, every function $f \in \mathscr{S}$ has an inverse map $f^{-1}$, defined by

$$
f^{-1}(f(z))=z \quad(z \in \mathbb{U}),
$$

and

$$
f\left(f^{-1}(w)\right)=w \quad\left(|w|<r_{0}(f) ; r_{0}(f) \geq \frac{1}{4}\right) .
$$

In fact, the inverse function is given by

$$
f^{-1}(w)=w-a_{2} w^{2}+\left(2 a_{2}^{2}-a_{3}\right) w^{3}-\left(5 a_{2}^{3}-5 a_{2} a_{3}+a_{4}\right) w^{4}+\cdots .
$$

A function $f \in \mathscr{A}$ is said to be bi-univalent in $\mathbb{U}$ if both $f(z)$ and $f^{-1}(w)$ are univalent in $\mathbb{U}$. Let $\Sigma$ denote the class of bi-univalent functions in $\mathbb{U}$ given by (2.1). For a brief history and some intriguing examples of functions and characterization of the class $\Sigma$, see Srivastava et al. [21] and Frasin and Aouf [11], see also [2, 3, 4, 12, 15, 18].

The Chebyshev polynomials of the first and second kinds are orthogonal for $t \in[-1,1]$ and defined as follows: 
Definition 2.1. The Chebyshev polynomials of the first kind are defined by the following three-terms recurrence relation:

$$
\begin{aligned}
& T_{0}(t)=1, \\
& T_{1}(t)=t, \\
& T_{n+1}(t):=2 t T_{n}(t)-T_{n-1}(t) .
\end{aligned}
$$

The first few of the Chebyshev polynomials of the first kind are

$$
T_{2}(t)=2 t^{2}-1, T_{3}(t)=4 t^{3}-3 t, T_{4}(t)=8 t^{4}-8 t^{2}+1, \cdots
$$

The generating function for the Chebyshev polynomials of the first kind, $T_{n}(t)$, is given by:

$$
F(z, t)=\frac{1-t z}{1-2 t z+z^{2}}=\sum_{n=0}^{\infty} T_{n}(t) z^{n} \quad(z \in \mathbb{U}) .
$$

Definition 2.2. The Chebyshev polynomials of the second kind are defined by the following three-terms recurrence relation:

$$
\begin{aligned}
& U_{0}(t)=1 \\
& U_{1}(t)=2 t \\
& U_{n+1}(t):=2 t U_{n}(t)-U_{n-1}(t) .
\end{aligned}
$$

The first few of the Chebyshev polynomials of the second kind are

$$
U_{2}(t)=4 t^{2}-1, U_{3}(t)=8 t^{3}-4 t, U_{4}(t)=16 t^{4}-12 t^{2}+1, \cdots
$$

The generating function for the Chebyshev polynomials of the second kind, $U_{n}(t)$, is given by:

$$
H(z, t)=\frac{1}{1-2 t z+z^{2}}=\sum_{n=0}^{\infty} U_{n}(t) z^{n} \quad(z \in \mathbb{U}) .
$$

The Chebyshev polynomials of the first and second kinds are connected by the following relations:

$$
\frac{d T_{n}(t)}{d t}=n U_{n-1}(t) ; T_{n}(t)=U_{n}(t)-t U_{n-1}(t) ; 2 T_{n}(t)=U_{n}(t)-U_{n-2}(t) .
$$

Definition 2.3. For $\lambda \geq 1, \mu \geq 0$ and $t \in(1 / 2,1)$, a function $f \in \Sigma$ given by (2.1) is said to be in the class $\mathscr{B}_{\Sigma}(\lambda, \mu, t)$ if the following subordinations hold for all $z, w \in \mathbb{U}$ :

$$
(1-\lambda) \frac{f(z)}{z}+\lambda f^{\prime}(z)+\mu z f^{\prime \prime}(z) \prec H(z, t):=\frac{1}{1-2 t z+z^{2}}
$$

and

$$
(1-\lambda) \frac{g(w)}{w}+\lambda g^{\prime}(w)+\mu w g^{\prime \prime}(w) \prec H(w, t):=\frac{1}{1-2 t w+w^{2}},
$$

where the function $g(w)=f^{-1}(w)$ is defined by (2.2). 
Remark 2.4. (1) For $\lambda=1$ and $\mu=0$, we have the class $\mathscr{B}_{\Sigma}(1,0, t):=\mathscr{B}_{\Sigma}(t)$ of functions $f \in \Sigma$ given by (2.1) and satisfying the following subordination conditions for all $z, w \in \mathbb{U}$ :

$$
f^{\prime}(z) \prec H(z, t)=\frac{1}{1-2 t z+z^{2}}
$$

and

$$
g^{\prime}(w) \prec H(w, t)=\frac{1}{1-2 t w+w^{2}} .
$$

This class of functions have been introduced and studied by Altinkaya and Yalçin $[5]$.

(2) For $\mu=0$, we have the class $\mathscr{B}_{\Sigma}(\lambda, 0, t):=\mathscr{B}_{\Sigma}(\lambda, t)$ of functions $f \in \Sigma$ given by (2.1) and satisfying the following subordination conditions for all $z, w \in \mathbb{U}$ :

$$
(1-\lambda) \frac{f(z)}{z}+\lambda f^{\prime}(z) \prec H(z, t)=\frac{1}{1-2 t z+z^{2}}
$$

and

$$
(1-\lambda) \frac{g(w)}{w}+\lambda g^{\prime}(w) \prec H(w, t)=\frac{1}{1-2 t w+w^{2}} .
$$

This class of functions have been introduced and studied by Bulut et al. [7].

\section{Coefficient Bounds for the function Class $\mathscr{B}_{\Sigma}(\lambda, \mu, t)$}

We begin with the following result involving initial coefficient bounds for the function class $\mathscr{B}_{\Sigma}(\lambda, \mu, t)$.

Theorem 3.1. Let the function $f(z)$ given by (2.1) be in the class $\mathscr{B}_{\Sigma}(\lambda, \mu, t)$. Then

$$
\left|a_{2}\right| \leq \frac{2 t \sqrt{2 t}}{\sqrt{\left|(1+\lambda+2 \mu)^{2}-4 t^{2}\left[(\lambda+2 \mu)^{2}-2 \mu\right]\right|}}
$$

and

$$
\left|a_{3}\right| \leq \frac{4 t^{2}}{(1+\lambda+2 \mu)^{2}}+\frac{2 t}{1+2 \lambda+6 \mu}
$$

Proof. Let $f \in \mathscr{B}_{\Sigma}(\lambda, \mu, t)$. From (2.5) and (2.6), we have

$$
(1-\lambda) \frac{f(z)}{z}+\lambda f^{\prime}(z)+\mu z f^{\prime \prime}(z)=1+U_{1}(t) w(z)+U_{2}(t) w^{2}(z)+\cdots
$$

and

$$
(1-\lambda) \frac{g(w)}{w}+\lambda g^{\prime}(w)+\mu w g^{\prime \prime}(w)=1+U_{1}(t) v(w)+U_{2}(t) v^{2}(w)+\cdots,
$$

for some analytic functions

$$
w(z)=c_{1} z+c_{2} z^{2}+c_{3} z^{3}+\cdots \quad(z \in \mathbb{U}),
$$


and

$$
v(w)=d_{1} w+d_{2} w^{2}+d_{3} w^{3}+\cdots \quad(w \in \mathbb{U})
$$

such that $w(0)=v(0)=0,|w(z)|<1(z \in \mathbb{U})$ and $|v(w)|<1 \quad(w \in \mathbb{U})$.

It follows from (3.3) and (3.4) that

$$
(1-\lambda) \frac{f(z)}{z}+\lambda f^{\prime}(z)+\mu z f^{\prime \prime}(z)=1+U_{1}(t) c_{1} z+\left[U_{1}(t) c_{2}+U_{2}(t) c_{1}^{2}\right] z^{2}+\cdots
$$

and

$$
\left.(1-\lambda) \frac{g(w)}{w}+\lambda g^{\prime}(w)+\mu w g^{\prime \prime}(w)=1+U_{1}(t) d_{1} w+\left[U_{1}(t) d_{2}+U_{2}(t) d_{1}^{2}\right]\right) w^{2}+\cdots .
$$

A short calculation shows that

$$
\begin{gathered}
(1+\lambda+2 \mu) a_{2}=U_{1}(t) c_{1}, \\
(1+2 \lambda+6 \mu) a_{3}=U_{1}(t) c_{2}+U_{2}(t) c_{1}^{2},
\end{gathered}
$$

and

$$
\begin{gathered}
-(1+\lambda+2 \mu) a_{2}=U_{1}(t) d_{1}, \\
(1+2 \lambda+6 \mu)\left(2 a_{2}^{2}-a_{3}\right)=U_{1}(t) d_{2}+U_{2}(t) d_{1}^{2} .
\end{gathered}
$$

From (3.5) and (3.7), we have

$$
c_{1}=-d_{1},
$$

and

$$
2(1+\lambda+2 \mu)^{2} a_{2}^{2}=U_{1}^{2}(t)\left(c_{1}^{2}+d_{1}^{2}\right) .
$$

By adding (3.6) to (3.8), we get

$$
2(1+2 \lambda+6 \mu) a_{2}^{2}=U_{1}(t)\left(c_{2}+d_{2}\right)+U_{2}(t)\left(c_{1}^{2}+d_{1}^{2}\right) .
$$

By using (3.10) in (3.11), we obtain

$$
\left[2(1+2 \lambda+6 \mu)-\frac{2 U_{2}(t)}{U_{1}^{2}(t)}(1+\lambda+2 \mu)^{2}\right] a_{2}^{2}=U_{1}(t)\left(c_{2}+d_{2}\right) .
$$

It is fairly well known [9] that if $|w(z)|<1$ and $|v(w)|<1$, then

$$
\left|c_{j}\right| \leq 1 \text { and }\left|d_{j}\right| \leq 1 \text { for all } j \in \mathbb{N} .
$$

By considering (2.4) and (3.13), we get from (3.12) the desired inequality (3.1).

Next, by subtracting (3.8) from (3.6), we have 


$$
2(1+2 \lambda+6 \mu) a_{3}-2(1+2 \lambda+6 \mu) a_{2}^{2}=U_{1}(t)\left(c_{2}-d_{2}\right)+U_{2}(t)\left(c_{1}^{2}-d_{1}^{2}\right) .
$$

Further, in view of (3.9), it follows from (3.14) that

$$
a_{3}=a_{2}^{2}+\frac{U_{1}(t)}{2(1+2 \lambda+6 \mu)}\left(c_{2}-d_{2}\right)
$$

By considering (3.10) and (3.13), we get from (3.15) the desired inequality (3.2). This completes the proof of Theorem 3.1 .

Taking $\lambda=1$ and $\mu=0$ in Theorem 3.1, we get the following corollary.

Corollary 3.2. [7] Let the function $f(z)$ given by (2.1) be in the class $\mathscr{B}_{\Sigma}(t)$. Then

$$
\left|a_{2}\right| \leq \frac{t \sqrt{2 t}}{\sqrt{1-t^{2}}}
$$

and

$$
\left|a_{3}\right| \leq t^{2}+\frac{2}{3} t
$$

For Corollary 3.2, it's worthy to mention that Altinkaya and Yalçin [5] have obtained a remarkable result for the coefficient $\left|a_{2}\right|$, as shown in the following corollary.

Corollary 3.3. Let the function $f(z)$ given by (2.1) be in the class $\mathscr{B}_{\Sigma}(t)$. Then

$$
\left|a_{2}\right| \leq \frac{t \sqrt{2 t}}{\sqrt{1+2 t-t^{2}}}
$$

Taking $\mu=0$ in Theorem 3.1, we get the following corollary.

Corollary 3.4. [7] Let the function $f(z)$ given by (2.1) be in the class $\mathscr{B}_{\Sigma}(\lambda, t)$. Then

$$
\left|a_{2}\right| \leq \frac{2 t \sqrt{2 t}}{\sqrt{\left|(1+\lambda)^{2}-4 t^{2} \lambda^{2}\right|}}
$$

and

$$
\left|a_{3}\right| \leq \frac{4 t^{2}}{(1+\lambda)^{2}}+\frac{2 t}{1+2 \lambda}
$$

\section{Fekete-Szegö inequality for the function Class $\mathscr{B}_{\Sigma}(\lambda, \mu, t)$}

Now, we are ready to find the sharp bounds of Fekete-Szegö functional $a_{3}-\eta a_{2}^{2}$ defined for $f \in \mathscr{B}_{\Sigma}(\lambda, \mu, t)$ given by $(2.1)$. 
Theorem 4.1. Let the function $f(z)$ given by (2.1) be in the class $\mathscr{B}_{\Sigma}(\lambda, \mu, t)$. Then for some $\eta \in \mathbb{R}$,

$$
\left|a_{3}-\eta a_{2}^{2}\right| \leq\left\{\begin{array}{cl}
\frac{2 t}{1+2 \lambda+6 \mu}, & |\eta-1| \leq M \\
\frac{8|\eta-1| t^{3}}{\left|(1+\lambda+2 \mu)^{2}-4 t^{2}\left[(\lambda+2 \mu)^{2}-2 \mu\right]\right|}, & |\eta-1| \geq M
\end{array}\right.
$$

where

$$
M:=\frac{\left|(1+\lambda+2 \mu)^{2}-4 t^{2}\left[(\lambda+2 \mu)^{2}-2 \mu\right]\right|}{4(1+2 \lambda+6 \mu) t^{2}} .
$$

Proof. Let $f \in \mathscr{B}_{\Sigma}(\lambda, \mu, t)$. By using (3.12) and (3.15) for some $\eta \in \mathbb{R}$, we get

$$
\begin{aligned}
a_{3}-\eta a_{2}^{2} & =(1-\eta)\left[\frac{U_{1}^{3}(t)\left(c_{2}+d_{2}\right)}{2(1+2 \lambda+6 \mu) U_{1}^{2}(t)-2(1+\lambda+2 \mu)^{2} U_{2}(t)}\right]+\frac{U_{1}(t)\left(c_{2}-d_{2}\right)}{2(1+2 \lambda+6 \mu)} \\
& =U_{1}(t)\left[\left(h(\eta)+\frac{1}{2(1+2 \lambda+6 \mu)}\right) c_{2}+\left(h(\eta)-\frac{1}{2(1+2 \lambda+6 \mu)}\right) d_{2}\right],
\end{aligned}
$$

where

$$
h(\eta)=\frac{U_{1}^{2}(t)(1-\eta)}{2\left[(1+2 \lambda+6 \mu) U_{1}^{2}(t)-(1+\lambda+2 \mu)^{2} U_{2}(t)\right]} .
$$

Then, in view of (2.4), we easily conclude that

$$
\left|a_{3}-\eta a_{2}^{2}\right| \leq \begin{cases}\frac{2 t}{1+2 \lambda+6 \mu}, & |h(\eta)| \leq \frac{1}{2(1+2 \lambda+6 \mu)} \\ 4|h(\eta)| t, & |h(\eta)| \geq \frac{1}{2(1+2 \lambda+6 \mu)}\end{cases}
$$

This proves Theorem 4.1.

We end this section with some corollaries concerning the sharp bounds of Fekete-Szegö functional $a_{3}-\eta a_{2}^{2}$ defined for $f \in \mathscr{B}_{\Sigma}(\lambda, \mu, t)$ given by (2.1).

Taking $\eta=1$ in Theorem 4.1, we get the following corollary.

Corollary 4.2. Let the function $f(z)$ given by (2.1) be in the class $\mathscr{B}_{\Sigma}(\lambda, \mu, t)$. Then

$$
\left|a_{3}-a_{2}^{2}\right| \leq \frac{2 t}{1+2 \lambda+6 \mu}
$$

Taking $\lambda=1$ and $\mu=0$ in Theorem 4.1, we get the following corollary.

Corollary 4.3. Let the function $f(z)$ given by (2.1) be in the class $\mathscr{B}_{\Sigma}(t)$. Then for some $\eta \in \mathbb{R}$,

$$
\left|a_{3}-\eta a_{2}^{2}\right| \leq \begin{cases}\frac{2}{3} t, & |\eta-1| \leq \frac{1-t^{2}}{3 t^{2}} \\ \frac{2|\eta-1| t^{3}}{1-t^{2}}, & |\eta-1| \geq \frac{1-t^{2}}{3 t^{2}}\end{cases}
$$

Taking $\eta=1$ in Corollary 4.3, we get the following corollary. 
Corollary 4.4. Let the function $f(z)$ given be (2.1) be in the class $\mathscr{B}_{\Sigma}(t)$. Then

$$
\left|a_{3}-a_{2}^{2}\right| \leq \frac{2}{3} t
$$

Taking $\mu=0$ in Theorem 4.1, we get the following corollary.

Corollary 4.5. Let the function $f(z)$ given by (2.1) be in the class $\mathscr{B}_{\Sigma}(\lambda, t)$. Then for some $\eta \in \mathbb{R}$,

$$
\left|a_{3}-\eta a_{2}^{2}\right| \leq \begin{cases}\frac{2 t}{1+2 \lambda}, & |\eta-1| \leq \frac{\left|(1+\lambda)^{2}-4 t^{2} \lambda^{2}\right|}{4(1+2 \lambda) t^{2}} \\ \frac{8|\eta-1| t^{3}}{\left|(1+\lambda)^{2}-4 t^{2} \lambda^{2}\right|}, & |\eta-1| \geq \frac{\left|(1+\lambda)^{2}-4 t^{2} \lambda^{2}\right|}{4(1+2 \lambda) t^{2}}\end{cases}
$$

Taking $\eta=1$ in Corollary 4.5, we get the following corollary.

Corollary 4.6. Let the function $f(z)$ given by (2.1) be in the class $\mathscr{B}_{\Sigma}(\lambda, t)$. Then

$$
\left|a_{3}-a_{2}^{2}\right| \leq \frac{2 t}{1+2 \lambda}
$$

\section{REFERENCES}

[1] T. Al-Hawary, B. A. Frasin and M. Darus, Fekete-Szegö problem for certain classes of analytic functions of complex order defined by the Dziok-Srivastava operator, Acta Mathematica Vietnamica 39.2 (2014), 185-192.

[2] S. Altinkaya and S. Yalçin, Initial coefficient bounds for a general class of bi-univalent functions, International Journal of Analysis, Article ID 867871,(2014), 4 pp.

[3] Ş. Altinkaya and S. Yalçin, Coefficient bounds for a subclass of bi-univalent functions, TWMS Journal of Pure and Applied Mathematics, 6 (2) 2015.

[4] Ş. Altinkaya and S. Yalçin, Coefficient Estimates for Two New Subclasses of Bi-univalent Functions with respect to Symmetric Points, Journal of Function Spaces, (2015), Article ID 145242, 5 pages.

[5] Ş. Altinkaya and S. Yalçin, Estimates on coefficients of a general subclass of bi-univalent functions associated with symmetric q-derivative operator by means of the Chebyshev polynomials, Asia Pacific Journal of Mathematics, 4(2) (2017), 90-99.

[6] Ş. Altinkaya and S. Yalçin, On the Chebyshev polynomial coefficient problem of some subclasses of bi-univalent functions, Gulf Journal of Mathematics, (2017), 1464-1473.

[7] S. Bulut, N. Magesh and V. K. Balaji, Initial bounds for analytic and biunivalent functions by means of chebyshev polynomials, Analysis 11.1 (2017), 83-89.

[8] E. H. Doha, The first and second kind Chebyshev coefficients of the moments of the general-order derivative of an infinitely differentiable function, Int. J. of Comput. Math. 51 (1994), 21-35.

[9] P. L. Duren, Univalent functions, Grundlehren der Mathematischen Wissenschaften 259, SpringerVerlag, New York, 1983.

[10] J. Dziok, R. K. Raina and J. Sokol, Application of Chebyshev polynomials to classes of analytic functions, Comptes Rendus Mathematique 353.5 (2015), 433-438.

[11] B. A. Frasin and M. K. Aouf, New subclasses of bi-univalent functions, Appl. Math. Lett. 24, 9 (2011), 1569-1573.

[12] B. A. Frasin and Tariq Al-Hawary, Initial Maclaurin Coefficients Bounds for New Subclasses of Bi-univalent Functions, Theory and App. of Math. \& Computer Science 5 (2) (2015) 186-193.

[13] M. Fekete and G. Szegö, Eine Bermerkung über ungerade schlichte Funktionen, Journal of the London Mathematical Societ 1.2 (1933), 85-89. 
[14] H. Ö. Güney, G. Murugusundaramoorthy and K. Vijaya, Coefficient Bounds for Subclasses of Biunivalent Functions Associated with the Chebyshev Polynomials, Journal of Complex Analysis (2017), 11 pages.

[15] N. Magesh and J. Yamini, Coefficient bounds for a certain subclass of bi-univalent functions, International Mathematical Forum, 8 (27) (2013), 1337-1344.

[16] J. C. Mason, Chebyshev polynomial approximations for the L-membrane eigenvalue problem, SIAM J. Appl. Math. 15 (1967), 172-186.

[17] S. S. Miller and P. T. Mocanu, Differential Subordination: theory and applications, CRC Press, New York, 2000.

[18] S. Porwal and M. Darus, On a new subclass of bi-univalent functions, J. Egypt. Math. Soc., 21 (3) (2013), 190-193.

[19] B. Simon, Orthogonal polynomials on the unit circle, American Mathematical Society, 2009.

[20] H. M. Srivastava, A. K. Mishra, and M. K. Das, The fekete-szegö-problem for a subclass of close-toconvex functions, Complex Variables and Elliptic Equations 44.2 (2001), 145-163.

[21] H. M. Srivastava, A. K. Mishra and P. Gochhayat, Certain subclasses of analytic and bi-univalent functions, Appl. Math. Lett. 23 (2010), 1188-1192.

[22] G. Szegö, Orthogonal polynomials, American Mathematical Society, New York, 2003. Szeg, Gabor. Orthogonal polynomials. Vol. 23. American Mathematical Soc., 1939.

[23] F. Yousef, A. A. Amourah and M. Darus, Differential sandwich theorems for p-valent functions associated with a certain generalized differential operator and integral operator, Italian Journal of Pure and Applied Mathematics 36 (2016), 543-556.

Feras Yousef: Department of Mathematics, Faculty of Science, The University of JoRDAN, AMMAN 11942, JoRDAN.

E-mail address: fyousef@ju.edu.jo (Corresponding author)

B. A. Frasin: Faculty of Science, Department of Mathematics, Al al-Bayt University, MAFraq, JordAN.

E-mail address: bafrasin@yahoo.com

Tariq Al-Hawary: Department of Applied Science, Ajloun College, Al-Balqa Applied University, AJloun 26816, Jordan.

E-mail address: tariq_amh@yahoo.com 